EPJ Web of Conferences 71, 00136 (2014)

DOI: $10.1051 /$ epjconf / 20147100136

(C) Owned by the authors, published by EDP Sciences, 2014

\title{
Higgs Physics at CMS
}

\author{
Miguel Vidal ${ }^{1, a}$ for the CMS Collaboration \\ ${ }^{1}$ Centre for Cosmology, Particle Physics and Phenomenology - CP3, Université catholique de Louvain, \\ Louvain-la-Neuve, Belgium
}

\begin{abstract}
Searches for the Higgs boson at CMS, based on the data collected in 2011 and 2012 at 7 and $8 \mathrm{TeV}$ centre-of-mass energy, corresponding to integrated luminosities of 5 and $20 \mathrm{fb}^{-1}$, are presented. The observed new boson is found to be consistent with the Standard Model Higgs boson in all investigated properties such as the spin and couplings to bosons and fermions. The measured mass of this new particle is $125.7 \pm 0.3$ (stat.) \pm 0.3 (syst.) GeV.
\end{abstract}

\section{Introduction}

The Standard Model (SM) predicts the existence of a Higgs boson responsible for the spontaneous electroweak symmetry breaking [1, 2]. In July 2012, the ATLAS and CMS [3] Collaborations announced the discovery of a new boson $[4,5]$ with a mass around $126 \mathrm{GeV}$. From this moment one the highest priorities of the CMS Collaboration was to establish the nature of this new partible by studying its properties, i.e. observe its decay modes, measure its mass and couplings, and probe its spin-parity, relying on the full dataset that amounts to $5.1 \mathrm{fb}^{-1}$ at $7 \mathrm{TeV}$ and $19.6 \mathrm{fb}^{-1}$ at $8 \mathrm{TeV}$ centre-of-mass energy.

The most sensitive channels at LHC for the search of the Higgs boson are described in section 2, other channels, including less sensitive searches, scan over different mass regions, and beyond the SM interpretations, are summarised in section 3 and finally a summary is given in section 4 .

\section{SM Higgs Searches at $125 \mathrm{GeV}$}

The five most sensitive channels contributing to the discovery of the Higgs boson with mass of approximately $126 \mathrm{GeV}$ are presented in this section along with the properties of the new particle, extracted from the combination of the relevant channels in each case.

\section{$2.1 \mathrm{H} \rightarrow \mathrm{ZZ} \rightarrow 41$}

The four lepton channel provides the cleanest possible experimental signature, a peak in the four lepton mass spectrum on top of a flat and small background. The boson is observed in the search for the standard model Higgs boson in the mass range $110<\mathrm{m}_{\mathrm{H}}<1000 \mathrm{GeV}$ in the $\mathrm{H} \rightarrow \mathrm{ZZ}$

\footnotetext{
a e-mail: miguel.vidal.marono@cern.ch
} 
decay channel [6]. The search considers final states where both Z's decay to electron, muon, or tau lepton pairs. The analysis uses pp collision data recorded by the CMS detector at the LHC, corresponding to integrated luminosities of $5.1 \mathrm{fb}^{-1}$ at $\sqrt{s}=7 \mathrm{TeV}$ and $19.6 \mathrm{fb}^{-1}$ at $\sqrt{s}=8 \mathrm{TeV}$. It makes use of the measured four-lepton mass (figure 1), the mass uncertainty, kinematic discriminants, and information sensitive to the production mechanism, such as associated dijet characteristics and transverse momentum of the four-lepton system.

The analysis presented in this report relies critically on the reconstruction, identification, and isolation of leptons. The high lepton reconstruction efficiencies are achieved for a $\mathrm{ZZ}$ system composed of two pairs of same-flavour and opposite-charge isolated leptons, $\mathrm{e}^{+} \mathrm{e}^{-}, \mu^{+} \mu^{-}$, or $\tau^{+} \tau^{-}$, in the measurement range $\mathrm{m}_{41}, \mathrm{~m}_{212 \tau}>100 \mathrm{GeV}$. One or both of the $\mathrm{Z}$ bosons can be off-shell. The $\mathrm{Z} \rightarrow 41$ resonance is used in the mass range $70<\mathrm{m}_{41}<100 \mathrm{GeV}$ to cross-check our mass measurement method. The background sources include an irreducible four-lepton contribution from direct ZZ (or $\mathrm{Z}^{*}$ ) production via $\mathrm{q} \overline{\mathrm{q}}$ annihilation and gg fusion. Reducible contributions arise from $\mathrm{Zb} \overline{\mathrm{b}}$ and $\mathrm{t} \overline{\mathrm{t}}$ where the final states contain two isolated leptons and two $b$ jets producing secondary leptons. Additional background of instrumental nature arises from $\mathrm{Z}+$ jets, $\mathrm{Z}+\gamma+$ jets, and $\mathrm{WZ}+$ jets events where jets are misidentified as leptons.

The boson is observed in channels with electron and muon pairs with a local significance above the expected background of 6.7 standard deviations, as shown in figure 2, with the mass $125.8 \pm$ 0.5 (stat.) \pm 0.2 (syst.) $\mathrm{GeV}$. The signal strength $\mu$, relative to the expectation for the standard model Higgs boson, is measured to be $\mu=0.91_{-0.24}^{+0.30}$. The signal strength modifiers associated with vector bosons and fermions in production are measured to be $\mu_{V}=1.0_{-2.3}^{+2.4}$ and $\mu_{F}=0.9_{-0.4}^{+0.5}$ (figure 2), thus consistent with the standard model expectations. The spin-parity of the boson is studied and the pure scalar hypothesis is found to be consistent with the observation when compared to six other spinparity hypotheses. The data disfavour the pure pseudoscalar hypothesis 0 - with a CLs value of $0.16 \%$, and disfavour the pure spin- 2 hypothesis of a narrow resonance with the minimal couplings to the vector bosons with a CLs value of $1.5 \%$. The spin-1 hypotheses are disfavoured with an even higher confidence. No other significant standard model Higgs-like excess is found in the search and upper limits at $95 \%$ confidence level exclude the range $130-827 \mathrm{GeV}$.

\section{$2.2 \mathrm{H} \rightarrow \gamma \gamma$}

The $\mathrm{H} \rightarrow \gamma \gamma$ decay channel provides a clean final-state topology which allows the mass to be reconstructed with high precision. In the mass range $110<\mathrm{m}_{\mathrm{H}}<150 \mathrm{GeV}, \mathrm{H} \rightarrow \gamma \gamma$ is one of the most promising channels for the Higgs search at the LHC despite its low branching fraction varying between $0.14 \%$ and $0.23 \%$. The primary production mechanism of the Higgs boson at the LHC is gluon fusion with additional smaller contributions from vector boson fusion (VBF) and production in association with a $\mathrm{W}$ or $\mathrm{Z}$ boson, or with a ț pair.

The analysis searches for a localized excess of diphoton events over a smoothly falling background due to prompt diphoton production and to events with at least one jet misidentified as photon. To achieve the best sensitivity to a Standard Model Higgs boson decaying to two photons, the events are separated into classes. The search results are presented for an analysis that uses Multi-VariateAnalysis (MVA) techniques both for photon identification and event classification, and extracts the signal from the background using a fit to the diphoton mass spectrum (referred to in the following as "mass-fit-MVA"). An independent analysis is also presented in which the photon identification and the events classification is cut-based and in which the background model is derived as before from a fit to the diphoton mass spectrum (referred as "cut-based") both showed in figure 3.

The most sensitive, MVA, analysis observes an excess of events at a mass of $125 \mathrm{GeV}$, with a local significance of $3.2 \sigma$ (standard deviations), where a local significance of $4.2 \sigma$ is expected from 


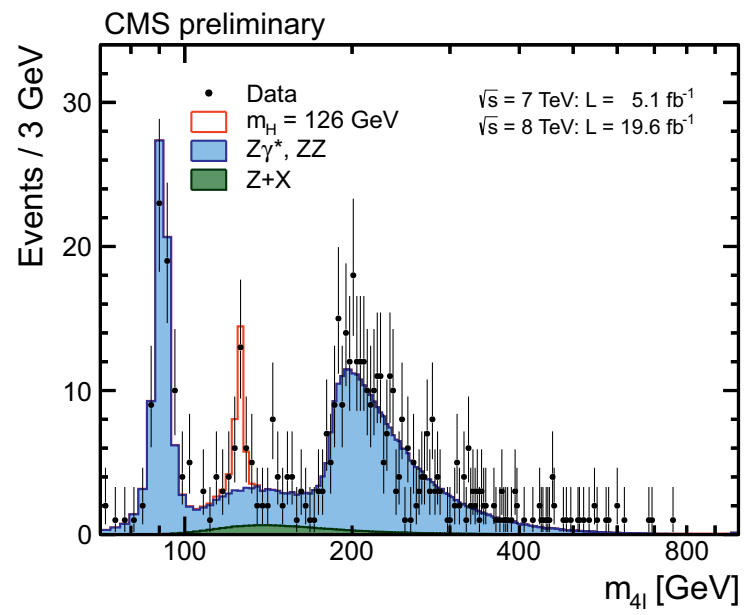

Figure 1. Distribution of the four-lepton reconstructed mass in for the sum of the $4 \mathrm{e}, 4 \mu$, and $2 \mathrm{e} 2 \mu$ channels. Points represent the data, shaded histograms represent the background and the unshaded histogram the signal expectation. The expected distributions are presented as stacked histograms. The measurements are presented for the sum of the data collected at $\sqrt{s}=7 \mathrm{TeV}$ and $\sqrt{s}=8 \mathrm{TeV}$. No event is observed for $\mathrm{m}_{41}>800 \mathrm{GeV}$.
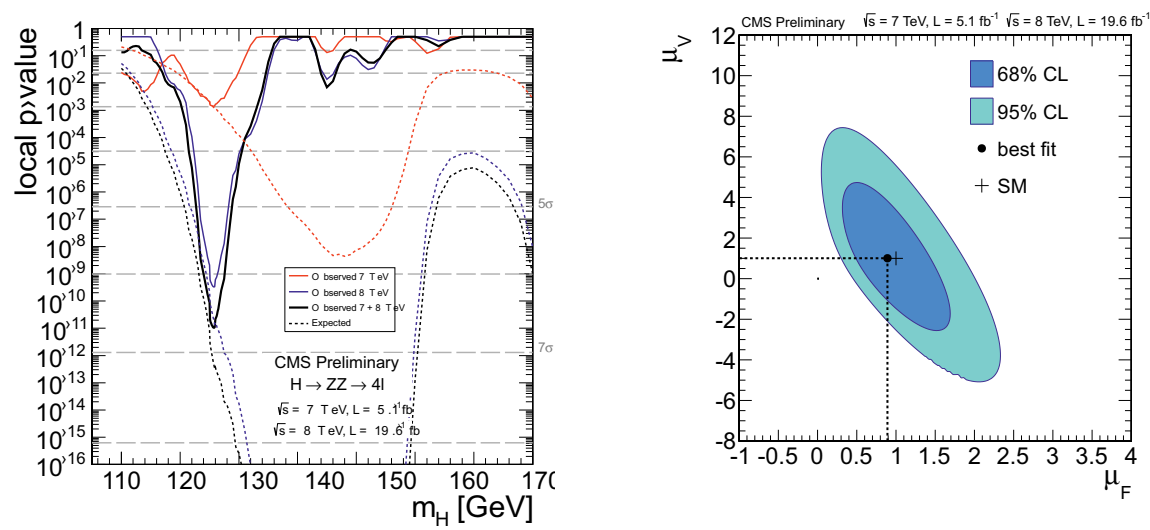

Figure 2. Significance of the local excess with respect to the standard model background expectation as a function of the Higgs boson mass for the $7 \mathrm{TeV}$ (red), $8 \mathrm{TeV}$ (blue) and $7 \mathrm{TeV}+8 \mathrm{TeV}$ (black) data sample. The results are shown for the full data sample in the range [110-180] GeV for the sum of the $4 \mathrm{e}, 4 \mu$, and $2 \mathrm{e} 2 \mu$ channels (left). Likelihood contours on the signal strength modifiers associated with fermions $\left(\mu_{F}\right)$ and vector bosons $\left(\mu_{V}\right)$ shown at $68 \%$ and $95 \%$ CL. (right)

a standard model Higgs boson. The best-fit signal strength, $\sigma / \sigma_{S M}$, is $0.78 \pm 0.27$ at $\mathrm{m}_{\mathrm{H}}=125 \mathrm{GeV}$, and the mass is fitted to be $125.4 \pm 0.5$ (stat.) \pm 0.6 (syst.) GeV. The cut-based analysis observes a corresponding excess with a local significance of $3.9 \sigma(3.5 \sigma$ expected $)$, and $\sigma / \sigma_{\mathrm{SM}}=1.11 \pm 0.31$ at 
$\mathrm{m}_{\mathrm{H}}=124.5 \mathrm{GeV}$. All measurements are compatible among themselves and with a Higgs boson with a mass of $125.4 \mathrm{GeV}$.
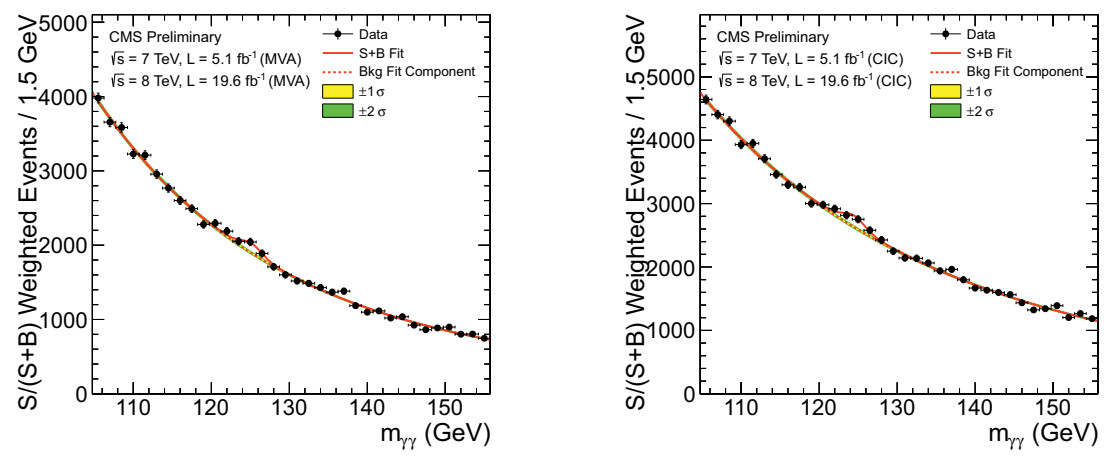

Figure 3. The diphoton invariant mass distribution with each event weighted by the $S /(S+B)$ value of its category, for the mass-fit-MVA (left) and cut-based (right) analyses on the 7 and $8 \mathrm{TeV}$ data sets combined. The lines represent the fitted background and signal, and the coloured bands represent the \pm 1 and \pm 2 standard deviation uncertainties in the background estimate.

\section{$2.3 \mathrm{H} \rightarrow \mathrm{WW}^{*} \rightarrow 212 v$}

This section describes the search for a Higgs boson decaying in a pair of $\mathrm{W}$ bosons in the CMS detector at $\sqrt{s}=7$ and $8 \mathrm{TeV}$. Events are classified according the exclusive jet multiplicity: 0, 1 . The events are further separated in same-flavor and different-flavor final states in each jet multiplicity. The main backgrounds $\left(\mathrm{W}^{+} \mathrm{W}^{-}\right.$, top, Drell Yan, $\left.\mathrm{W}+\mathrm{jets}\right)$ are estimated with data-driven techniques. The uncertainty on the background normalization represents the largest source of systematics of the analysis, together with the theoretical uncertainties on the Higgs cross section.

A cut and count analysis is performed, optimized for each mass point in all jet multiplicity categories. The same-flavor final states have limited sensitivity to the signal and introduce a large systematic uncertainty due to the large fake missing $\mathrm{E}_{\mathrm{T}}$ background in events with high pile-up. A 2D shape analysis of the di-leplon invariant and transverse masses for the different-flavor final state which increases the sensitivity to the standard model Higgs boson is performed and combined with the cut and count analysis in the same-flavor final state.

The search discussed here is performed over the mass range 110-600 GeV, and the data sample corresponds to 4.9 and $19.5 \mathrm{fb}^{-1}$ of integrated luminosity collected at center-of-mass energy of 7 and $8 \mathrm{TeV}$, respectively. An excess of events is observed above background which is consistent with the expectations from a standard model Higgs boson of mass $125 \mathrm{GeV}$, corresponding to an observed (expected) significance for $\mathrm{m}_{\mathrm{H}}=125 \mathrm{GeV}$ in terms of standad deviations of 4.0 (5.1), and a best fit value of the signal strength for $m_{H}=125 \mathrm{GeV}$ of $0.76 \pm 0.21$, as shown in figure 4 .

No other excess of events is observed over the full accessible mass range. Additional standard model Higgs-like bosons are excluded in the mass range $128-600 \mathrm{GeV}$ at $95 \%$ confidence level.

The 2D shape analysis of the di-leplon invariant and transverse masses for the different-flavor final state allows as well to perform an hypothesis separation test between the standard model Higgs 
boson for quantum numbers and couplings against the spin-2 hypothesis of a narrow resonance produced through the gluon fusion mechanism and with minimal couplings to the $W^{+} W^{-}$pair. Assuming the best fit value of the signal strength obtained for the individual hypothesis, the observed separation is 0.5 and 1.3 standard deviations away from the expected values for the standard model Higgs hypothesis and the spin-2 hypothesis, respectively.
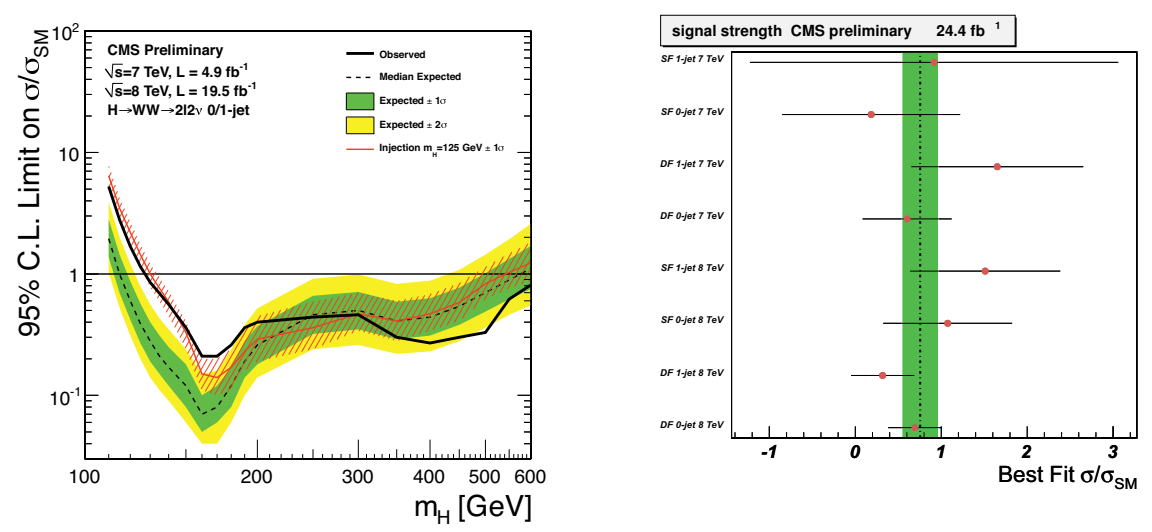

Figure 4. Expected and observed 95\% CL upper limits on the cross section times branching fraction, relative to the SM Higgs expectation, for the shape-based approach using the $7+8 \mathrm{TeV}$ data. For the shape-based approach, we combine the analysis in the different-flavor final state in the 0 -jet and 1-jet categories with the cut-based analysis in all other categories. The expected limits in the presence of the Higgs with $\mathrm{m}_{\mathrm{H}}=125 \mathrm{GeV}$ and its associated uncertainty are also shown (left). Best fit value of the signal strength $(\mu)$ for the shape-analysis for each channel.

\section{$2.4 \mathrm{VH}(\mathrm{b} \bar{b})$}

At a mass of $125 \mathrm{GeV}$ the standard model Higgs boson decays predominantly into a bottomantibottom quark pair $(b \bar{b})$. The observation and study of the $\mathrm{H} \rightarrow \mathrm{b} \bar{b}$ decay is therefore essential in determining the nature of the newly discovered boson. The measurement of $\mathrm{H} \rightarrow \mathrm{b} \overline{\mathrm{b}}$ will be the first direct test of whether the observed boson interacts as expected with the quark sector, as the coupling to the top quark has, so far, only been indirectly observed through loop-level interactions.

A search for the standard model Higgs boson decaying to $b \bar{b}$, when produced in association with a weak vector boson $(V)$, is reported for the following modes: $\mathrm{W}($ munu $) \mathrm{H}, \mathrm{W}(\mathrm{enu}) \mathrm{H}, \mathrm{W}(\mathrm{taunu}) \mathrm{H}$, $\mathrm{Z}$ (mumu) $\mathrm{H}, \mathrm{Z}(\mathrm{ee}) \mathrm{H}$ and $\mathrm{Z}$ (nunu) $\mathrm{H}$. The search is performed in data samples corresponding to integrated luminosities of $5.0 \mathrm{fb}^{-1}$ at $\sqrt{s}=7$ and up to $19.0 \mathrm{fb}^{-1}$ at $\sqrt{s}=8 \mathrm{TeV}$, recorded by the CMS experiment at the LHC. Upper limits, at the $95 \%$ confidence level, on the $\mathrm{VH}$ production cross section times the $\mathrm{H} \rightarrow \mathrm{b} \bar{b}$ branching ratio, with respect to the expectations for a standard model Higgs boson, are derived for a Higgs boson in the mass range 110-135 GeV. In this range, the observed upper limits vary from 1.1 to 3.1 times the standard model prediction; the corresponding expected limits vary from 0.7 to 1.5 (figure 5). At a Higgs boson mass of $125 \mathrm{GeV}$ the observed limit is 1.89 and the expected limit is 0.95 . An excess of events is observed above the expected background with a local significance of 2.1 standard deviations, which is consistent with the expectation from the production of the 
standard model Higgs boson. The signal strength corresponding to this excess, relative to that of the standard model Higgs boson, is $1.0 \pm 0.5$, as shown in figure 5 .
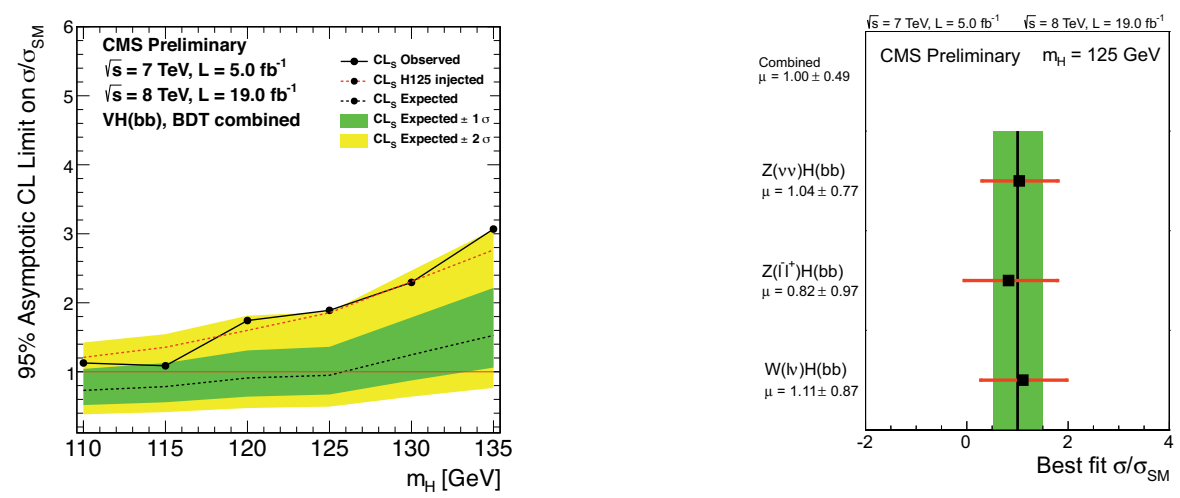

Figure 5. Expected and observed 95\% CL upper limits on the product of the VH production cross section times the Hbb branching ratio, with respect to the expectations for a standard model Higgs boson. The median expected limit and the 1- and 2-sigma bands are obtained with the LHC CLs method as implemented in RooStats, as are the observed limits at each mass point. The limits are combined for the $20117 \mathrm{TeV}$ and the $20128 \mathrm{TeV}$ data. The red dashed line represents the expected observed limits obtained from replacing the data with the sum of expected background and signal for a Higgs boson at a mass of $125 \mathrm{GeV}$ (left). The most likely value of the production cross section for a $125 \mathrm{GeV}$ Higgs boson, relative to the standard model cross section, for each channel and for all channels combined (right).

A search for the standard-model Higgs boson decaying to tau-lepton pairs is performed using events recorded by the CMS experiment at the LHC in 2011 and 2012 at a center-of-mass energy of 7 and $8 \mathrm{TeV}$ respectively. The dataset corresponds to an integrated luminosity of $4.9 \mathrm{fb}^{-1}$ of data taken at $7 \mathrm{TeV}$ center-of-mass energy and $19.4 \mathrm{fb}^{-1}$ at $8 \mathrm{TeV}$. The tau-lepton pair invariant-mass spectrum is studied in five different final states corresponding to the decay modes of the two tau leptons.

Five independent $\tau$-pair final states are studied: $\mu \tau_{h}, e \tau_{\mathrm{h}}, \mathrm{e} \mu, \tau_{\mathrm{h}} \tau_{\mathrm{h}}$, and $\mu \mu$, where $\tau_{\mathrm{h}}$ denotes a reconstructed hadronic $\tau$ decay. In each channel, the signal is separated from the background, and in particular from the irreducible $\mathrm{Z} \rightarrow \tau \tau$, using the $\tau$-pair mass $\mathrm{m}_{\tau \tau}$ reconstructed from the fourmomenta of the visible decay products of the two $\tau$ leptons and from the missing transverse energy.

Events are classified by the number of additional jets in the final state to enhance the contribution of different Higgs boson production mechanisms. The zero- jet category is only used to constrain background normalization, identification efficiencies, and energy scales. The one-jet category selects primarily signal events with a Higgs boson produced by gluon fusion, or in association with a $\mathrm{W}$ or $\mathrm{Z}$ boson decaying hadronically. Events in the vector-boson fusion (VBF) category are required to have two jets separated by a large rapidity gap, which mainly selects signal events with a Higgs boson produced by vector-boson fusion and strongly enhances the signal contribution. 
We observe an excess of events over a broad $\mathrm{mH}$ range, with a maximum local significance of 2.94 sigma at $\mathrm{m}_{\mathrm{H}}=120 \mathrm{GeV}$, compatible with the presence of a standard-model Higgs boson with a mass around $125 \mathrm{GeV}$.
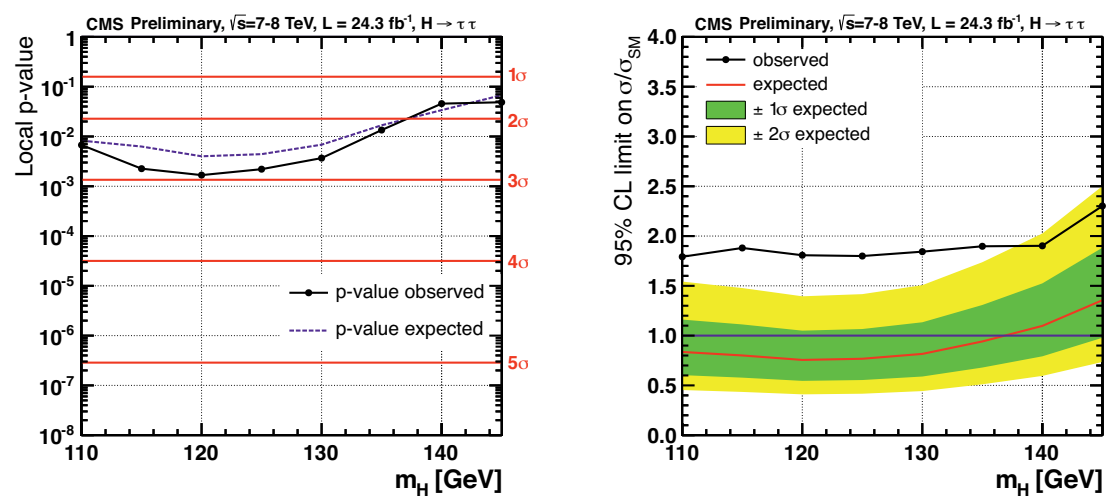

Figure 6. Observed and expected p-value 1-CLb, and the corresponding significance in number of standard deviations. These results include the search for a SM Higgs boson decaying into a tau pair and produced in association with a W or Z boson decaying leptonically (left). Combined observed 95\% CL upper limit on the signal strength parameter $\mu=\sigma / \sigma_{\mathrm{SM}}$, together with the expected limit obtained in the background hypothesis. These results include the search for a SM Higgs boson decaying into a tau pair and produced in association with a $\mathrm{W}$ or $\mathrm{Z}$ boson decaying leptonically (right).

\subsection{Properties}

Measurements of the properties of the recently discovered boson with a mass near $125 \mathrm{GeV}$ are presented [11]. The results are based on data samples corresponding to integrated luminosities of up to $5.1 \mathrm{fb}^{-1}$ at $7 \mathrm{TeV}$ and up to $19.6 \mathrm{fb}^{-1}$ at $8 \mathrm{TeV}$ in proton proton collisions at the LHC. Five decay modes are studied: $\gamma \gamma, \mathrm{ZZ}, \mathrm{WW}, \tau \tau$, and $b \bar{b}$. The mass of the new boson is measured to be $125.7 \pm 0.3$ (stat.) \pm 0.3 (syst.) $\mathrm{GeV}$. The event yields obtained by the different analyses targeting specific decay modes and production mechanisms are consistent with those expected for the standard model Higgs boson. The best-fit signal strength for all channels combined, expressed in units of the SM Higgs boson cross section, is $0.80 \pm 0.14$ at the measured mass, as shown in figure 7 . The consistency of the couplings of the observed boson with those predicted for the SM Higgs boson is tested in various ways, and no significant deviations are found (figure 7).

\section{Other Higgs Searches}

The wide Higgs program at CMS is not only performing searches for the five most sensitives channels were the new particle was discovered. Other Higgs searches are carried out to probe other production mechanisms, to cover the full mass range accessible by the CMS detector, and also trying to search for the Higgs particle in beyond the SM scenarios. Some of these analyses are summarised in this section. 

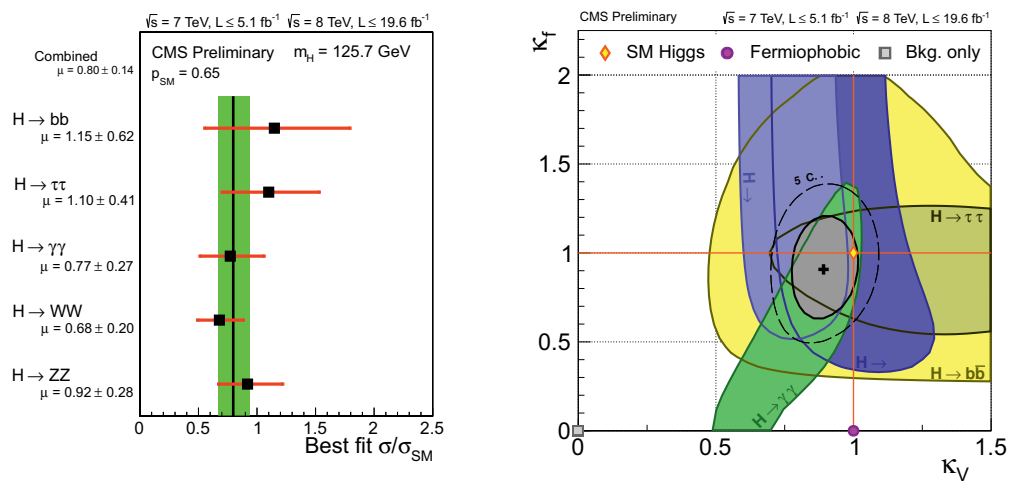

Figure 7. Higgs boson signal strength measured in the most sensitive channels. The line and the band indicate the overall fitted $\mu$ and its uncertainty (left). Confidence level contours of the scaling factors of the fermion couplings and the vector couplings of the Higgs boson. The contributions from the five different channels are also indicated (right).

\section{1 $\mathrm{ttH}$ production}

A search for the standard model Higgs boson produced in association with a top-quark pair in $19.5 \mathrm{fb}^{-1}$ of $8 \mathrm{TeV}$ pp collision data is reported [12]. The analysis is optimised for $\mathrm{ttH}$ events which are categorised into three channels based on the different final states: lepton + jets channel $\left(\mathrm{t} \overline{\mathrm{t}} \rightarrow \mathrm{l} v \mathrm{~b} \overline{\mathrm{b}} \mathrm{q} \overline{\mathrm{q}}, \mathrm{H} \rightarrow \mathrm{b} \overline{\mathrm{b}}\right.$ ), dilepton channel $\left(\mathrm{t} \overline{\mathrm{t}} \rightarrow 1^{+} \nu \mathrm{l}^{-} \nu \mathrm{b} \overline{\mathrm{b}}, \mathrm{H} \rightarrow \mathrm{b} \overline{\mathrm{b}}\right)$ and $\tau$ channel $(\mathrm{t} \overline{\mathrm{t}} \rightarrow \mathrm{l} v \mathrm{~b} \overline{\mathrm{b}} \mathrm{q} \overline{\mathrm{q}}, \mathrm{H} \rightarrow \tau \tau)$. The major background to the signal is top-quark pair production with additional jets. Multivariate analysis (MVA) techniques are used to discriminate between background and signal events. A simultaneous fit for signal and background is performed using the MVA output distributions. Assuming SM Higgs boson branching fractions, a 95\% C.L. upper limit on the ttH production cross section is presented.

A different search for ttH production, using for the first time the events where the Higgs boson decays to two photons, is also performed [13]. To maximize acceptance and sensitivity to such a small signal, we device two different sets of event selection criteria, optimized for leptonic and hadronic topantitop decays. We analyze $19.6 \mathrm{fb}^{-1}$ of $8 \mathrm{TeV}$ pp collisions and find no significant excess over the background-only predictions. We thus proceed to set an observed (expected) $95 \%$ confidence level upper limit on the top-antitop-Higgs production cross section times $\mathrm{BR}(\mathrm{H} \rightarrow \gamma \gamma)$ of 5.4 (5.3) times the Standard Model value, corresponding to $1.6 \mathrm{fb}$, for a Higgs boson of mass $\mathrm{m}_{\mathrm{H}}=125 \mathrm{GeV}$.

In addition, the results from both analyses are combined since they are independent.

\section{2 $\mathrm{H} \rightarrow \mathrm{ZZ}$ high mass}

A combination of the results showed in section 2.1 along with the results from the search for $\mathrm{H} \rightarrow \mathrm{ZZ} \rightarrow 212 v$ [14] and $\mathrm{H} \rightarrow \mathrm{ZZ} \rightarrow 2 \mathrm{l} 2 \mathrm{q}$ [15] are shown here. This combination covers the Higgs mass range from $200 \mathrm{GeV}$ to $1000 \mathrm{GeV}$. No deviations from the Standard Model expectation is observed as shown in figure 8 . 


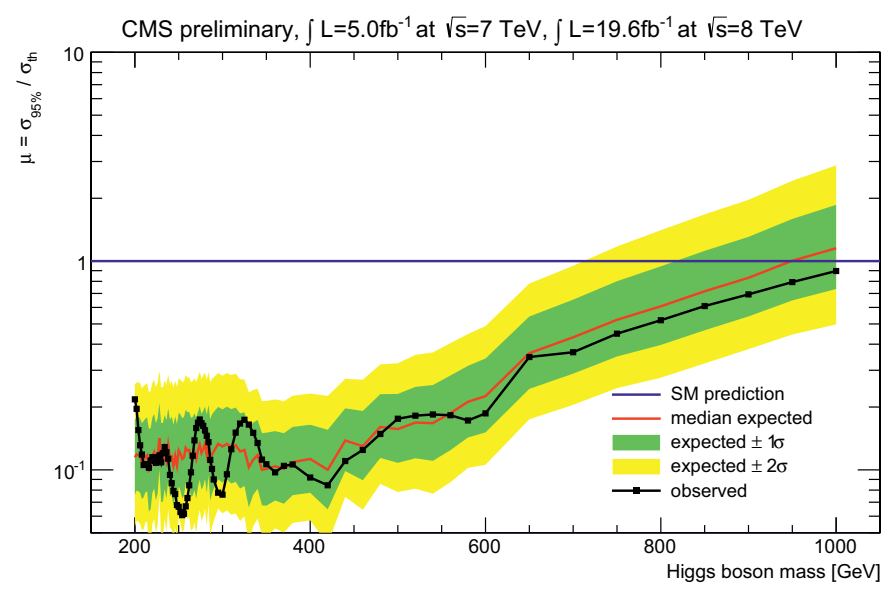

Figure 8. Observed (black line) and expected (red line) 95\% CL upper limit on the ratio of the product of the production cross section and branching fraction to the SM expectation for the Higgs boson decaying into two $\mathrm{Z}$ bosons obtained using the asymptotic CLs technique. The $68 \%$ and $95 \%$ CL ranges of expectation for the background-only model are also shown with green and yellow bands, respectively. The horizontal solid line at unity indicates the SM expectation. Result is combination of all individual channels: $\mathrm{H} \rightarrow \mathrm{ZZ} \rightarrow 2 \mathrm{l} 21$, $\mathrm{H} \rightarrow \mathrm{ZZ} \rightarrow 212 v$ and $\mathrm{H} \rightarrow \mathrm{ZZ} \rightarrow 212 \mathrm{q}$.

\subsection{Higgs to invisible}

A direct search for a standard-model-like Higgs boson produced in association with a $\mathrm{Z}$ boson and decaying to invisible particles is reported [16]. A non-zero partial decay width to invisible particles could provide evidence for physics beyond the standard model. The search is performed in data samples corresponding to integrated luminosities of $5.1 \mathrm{fb}^{-1}$ at $\sqrt{s}=7 \mathrm{TeV}$ and $19.6 \mathrm{fb}^{-1}$ at $\sqrt{s}=$ $8 \mathrm{TeV}$, recorded by the CMS experiment. No deviation from the background expectation is observed and limits are set on the branching fraction of the standard model Higgs boson decaying to invisible particles assuming the standard model production rate. For a Higgs boson with $\mathrm{m}_{\mathrm{H}}=125 \mathrm{GeV}$, the observed (expected) 95\% confidence level upper limit on the branching fraction of the Higgs boson to invisible particles is $75 \%(91 \%)$.

\section{Summary}

Preliminary results of the five most sensitive channels for the Higgs boson search around $125 \mathrm{GeV}$ have been reported. The full dataset collected at LHC in 2011 and 2012 at 7 and $8 \mathrm{TeV}$ centre-of-mass energy and corresponding to integrated luminosities of 5 and $20 \mathrm{fb}^{-1}$ have been used. The observed new boson with a measured mass of about $125.7 \pm 0.3$ (stat.) \pm 0.3 (syst.) $\mathrm{GeV}$ is found to be consistent with the Standard Model Higgs boson in all investigated properties. Different searches for beyond the SM Higgs bosons and non dominant production mechanisms have also been carried out and improved exclusion limits have been obtained. 


\section{References}

[1] P. W. Higgs, Phys. Rev. Lett. 13 (1964) 508

[2] F. Englert and R. Brout, Phys. Rev. Lett. 13 (1964) 321.

[3] CMS Collaboration, JINST 3 (2008) S08004

[4] ATLAS Collaboration, Phys. Lett. B 716, (2012) 1-29

[5] CMS Collaboration, Phys. Lett. B 716 (2012) 30-61

[6] CMS Collaboration, CMS Physics Analysis Summary HIG-13-002 (2013)

[7] CMS Collaboration, CMS Physics Analysis Summary HIG-13-001 (2013)

[8] CMS Collaboration, CMS Physics Analysis Summary HIG-13-003 (2013)

[9] CMS Collaboration, CMS Physics Analysis Summary HIG-13-012 (2013)

[10] CMS Collaboration, CMS Physics Analysis Summary HIG-13-004 (2013)

[11] CMS Collaboration, CMS Physics Analysis Summary HIG-13-005 (2013)

[12] CMS Collaboration, CMS Physics Analysis Summary HIG-13-019 (2013)

[13] CMS Collaboration, CMS Physics Analysis Summary HIG-13-015 (2013)

[14] CMS Collaboration, CMS Physics Analysis Summary HIG-13-014 (2013)

[15] CMS Collaboration, CMS Physics Analysis Summary HIG-12-024 (2013)

[16] CMS Collaboration, CMS Physics Analysis Summary HIG-13-018 (2013) 